\title{
Caring for social complexity in nanomedicine
}

In this paper, I will discuss from a 'Science and Technology Studies' perspective three different modes of caring about the social complexity in biomedical and nanomedical research. Nanomedical research unfolds a variety of issues that generate different concerns, questions, problems, requirements and interests that connect with different systems of action (in vitro, in vivo), different kinds (human, nonhuman) and different scales of action (nano, micro, macro). To adequately address the social complexity, I will discuss three possible modes of caring about social complexity: laboratory experiment and scientific analysis, public expert controversies, and publics. These different modes of caring share an experimental ethos that engages nanomedical issues for which no common solutions are available.

Keywords: care $\bullet$ culture of promise $\bullet$ experimentation $\bullet$ nanomedicine $\bullet$ public controversy - publics • social complexity

\section{Social complexity, care}

\section{\& experimentation}

"There is no creation without experiment" [1].

In this paper, I will discuss from a Science and Technology Studies perspective three modes of caring about social complexity in nanomedical research. Social complexity as understood here is not a given qualification that is simply found in nanoscaled relations. Social complexity refers to a particular set of experimental relations that arise from a bioand nanomedical perspective on issues of health and illness. It creates possibilities and problems, questions, objects and practices which remain often uncertain and only partially understood. What makes nanomedical research socially complex is precisely that it does not refer to a unilinear and smooth process from bench to bedside (see also Barz, Luxenhofer and Guchet, in this issue [2-4]). Rather, it unfolds a social topology of issues that generate a variety of different concerns, questions, problems, requirements and interests that connect with different systems of action (in vitrolin vivo), different kinds (human, nonhuman) and different scales of action (nano, micro, macro). Social complexity in nanomedicine can be understood as a process of multiple forms of translation: first, the translation of poorly solved or unresolved healthcare issues into biomedical ideas involving the nanoscale. Second, these ideas may translate into experimental laboratory settings that assemble novel nanoscale relations (biochemical, biophysical, magnetic, etc.), which, third, translate into novel smallscale in vitro relations and into in vivo experimentation. Fourth, in vivo tested experimental systems require the translation into the different interactions and environments of (highly individual) human bodies, clinical practices and concrete situations of medical intervention. Fifth, clinical results may then possibly translate into marketable and sustainable healthcare technologies and related practices. Moreover, these different stages have multidirectional effects on the translation process and thus add social complexity to it (see Pierce, the second part of this special focus issue [5]). Generally speaking, nanomedical research processes gain complexity since they engage with and produce
Michael Schillmeier

Department of Sociology, Philosophy \& Anthropology, Centre for the Studies of Life Sciences, University of Exeter, Byrne House, Exeter EX4 4PJ, UK Tel.: +44(0) 1392725125 m.schillmeier@exeter.ac.uk 
networks of new relations that shape and reshape what has been related - for the good or bad. These novel relations between different processes, objects and their environments are often only partly understood, contested and vividly disputed.

Nanomedical complexity is not confined to lab experiments. It potentially will affect vulnerable human bodies, change how we understand health and illness, modify animals into research models and reconfigure human/nonhuman relations, alter scientific organization, restructure clinical and other healthcare practices, produce issues of affordability, equal distribution and accessibility of nanomedicine and nanotechnologies and so forth [6-13]. These crossing effects will require and obligate practices of care that engage with the different 'matters of concern' and issues that arise thereby [14-17]. Caring about social complexity in nanomedicine, then, does not mean to divide between the domain of 'science' on the one hand and the different domains of 'society' by which scientific research is exposed to critical assessment on the other. Rather than an abstract aim of 'science meets society', it is the communication between all the different actors, their practices and concrete issues that is of crucial concern. It is the interface of the different matters of concern that come to the fore and articulates the issues that need to be cared for. Practices of care involve all relevant actors that are affected by nanomedical research. It includes scientists, scientific objects, patients, clinicians, technicians, concerned citizens, policy makers, philosophers, regulators, pharmaceutical enterprises, nongovernmental organizations and so on, as well as new actors emerging from these practices of care. However, practices of care also need to respect those who do not want to be involved.

From the experiences of laboratory experimentation, we can learn that caring practices are always about specific problems and their complexities as they emerge from particular objects of concern for which no shared solutions are available. In the following, I will discuss three sets of practices which enunciate most relevant practices of care that are required to ensure that nanomedical research enables good scientific practices. Good scientific practice not only cares about its own complex issues as they emerge within the laboratory settings, but involves a learning process of engaging with the relevant matters of concern and related actors - scientific and nonscientific, human and nonhuman.

Caring about the social complexity of nanomedicine and biomedical research in general evokes controversies and names novel forms of collective experimentations that cannot be reduced to the secluded laboratory, but at the same time, cannot do without it. We can learn from laboratory practices that the experiment is the strongest tool of scientific practice. Hence, it needs to be cared for in order to provide the conditions of possibility of the well-being of current scientific research in general and nanomedical research in particular.

Crucially, caring about social complexity in nanomedicine entails to have or acquire social competences as good practitioners do when they have to deal with a demanding and unsolved situation. Social competence as understood here refers to the ability to engage with the other and his/her/its practices which are not necessarily shared practices and thus allude to diverging perspectives and sometimes opposing interests, objectives and aims. It also means, as Bruno Latour has put, to engage in diplomatic practices, that is, "to learn to speak well to someone about something that really matters to that person" [18]. Caring about complexity adheres to problematize the idea "that there is only one way to judge truth and falsity" [19]. To care about social complexity multiplies the concerns relevant to a specific scientific endeavor in order to enable to communicate the requirements of, for example, nanoparticles, targeted drug delivery systems and other nanoformulations that have cross-scaling and cross-domain effects relating scientific practices with other forms of societal organization (as outlined above).

In the following, I will present and discuss different forms of practices that require caring for social complexity in nanomedical research enquiries. Following our understanding of social complexity which 'sets out problems' for which no solutions are ready at hand, the discussion will highlight three clusters of problems and related practices and locations [20]:

- Laboratory experimentation and scientific analysis;

- Public expert controversies;

- Publics.

Obviously, these modes of caring offer only a selection of possibilities and should not be read as a comprehensive list.

\section{Laboratory experimentation \& scientific analysis}

"The world is a laboratory, outside as well as inside." [21].

The culture of promise \& nanomedical research Caring about social complexity in nanomedicine is caring about prevailing problems we can find in current biomedical research activities that try to engage nanoscaled properties. One major problem in nanomedicine is the prevalent asymmetry between what I like to call the 'culture of promise' of applying nano- 
technologies and how nanomedical research lives up and is tied up to this promise.

The popular understanding of nanomedicine refers to nanomedicine as the application of the nanotechnological promise to healthcare [22-26]. Thereby, the nanotechnological promise is governed by the following assumption: The better we imitate/mimic 'nature' (as a given nanocomplex) the more we become able to produce human-designed artefacts (nanotechnologies, smart biocompatible drugs, etc.) that go beyond nature's capacities of creating sustainable modes of ordering. As a consequence, we will be capable to improve our abilities to diagnose, treat and cure illnesses and enhance mental, embodied and social life $[27,28]$. The promise is, for example, increasing effectiveness, safety and patient adherence, reducing healthcare costs and a significant contribution to the economy. If we are able to imitate nature's evolution in a way that is more than nature is able to offer, then, anything is possible. This is the radical claim that is at the heart of the nanotechnical promise [29].

Applied to medical issues, this promise has produced fairly utopian ideas of molecular machines, which in their essence aim at erasing illness and secure a permanent healthy state of human bodies throughout their lives. In 2004, the US National Cancer Institute announced the possibility of curing cancer by 2015 with the help of nanomedicine [30]. Or as RA Freitas Jr wrote in 1999: "Nanomedicine will involve designing and building a vast proliferation of incredibly efficacious molecular devices, and then deploying these devices in patients to establish and maintain a continuous state of human healthiness. (...) $[\mathrm{N}]$ anomedicine will employ molecular machine systems to address medical problems, and will use molecular knowledge to maintain human health at the molecular scale." [31].

Such claims do not reflect in any serious way the state of the art of current nanomedical research, but they do fit very well - although in alleviated forms with the culture of promise that accompanies nanomedical research today. Once enmeshed in the everyday affairs of nanomedical research, the real-time accomplishments are often fairly moderate and the general nanotechnological promise remains a promise. Having said this, the point I try to raise is not to neglect the productive developments within the diverse fields of nanomedical research, its potentials and its visions. This issue next to many exciting developments in nanomedicine provides a wide range of intriguing possibilities to innovate nanomedical research $[22,32-$ 35]. What I like to highlight instead is that a too big of a mismatch between promise and actual state of affairs in nanomedical research is counterproductive and may have negative effects for nanomedical research itself [36]. The asymmetry between promise and actuality sets out a problem that marks an on-going socially complex situation for nanomedical research.

For today, the nanotechnological promise holds insofar as it asks and legitimates ambitious future projects to explore a relatively unknown territory of nanoscaled processes and effects and develop techniques to characterize them. This includes the prospect of longer and more expensive preclinical and clinical research phases. At the same, this prospect faces timereduced research funding and a significant shortage of lab- and overall project-time to engage adequately in understanding nanoscaled relations. It also involves the recognition and assessment of overambitious and overcomplex projects. Clearly, nanomedical research includes much more than lab experiments as well as reproducible and applicable results: Writing grant applications and getting grants, delivering increasing quantities of publications in high impact journals, persistent visibility at conferences, obligatory networking with policy makers, regulators and nonuniversity partners (medical and pharma industry) as well as securing patents, investing in spin-offs and so forth. Nanomedical researchers are also meant to interact with the clinic, patients, interest groups and engage in auditable outreach projects. Communicating nanomedical research as a key mode of innovating healthcare became of considerable importance to strengthen societal acceptance of nanomedical research, to maintain and foster nanomedicine itself and related science policies of biomedical research agendas as well as to strengthen the pharma industry and regional, national and global economies.

All these activities may be considered as additives that burden and corrupt the 'purity' and 'autonomy' of scientific practices, although it seems obvious that the history of (modern) science has always been more than the changing conditions of the lab experiment [37-39]. Having said this, the enforced intermingling of scientific and nonscientific practices may indeed be disastrous if, for example, science is or will be defined by nonscientific practice alone (e.g., economic reasoning). This is the more of a concern in nanomedicine since it is considered to be 'applied research'. This is why - similar to the 1960 s - a quest for saving the 'autonomy' of science (including nanomedical research) frequently appears as a remedy for troubled scientific enterprises. Thus, to engage in experimentation outside the lab is prone to be considered as a nonscientific obligation that will not contribute to the progress of nanomedical research, but is easily dismissed as the squandering of limited research money since it keeps scientists from doing more important things in the lab. 
Fast science \& laboratory requirements

Clearly, the culture of promise of applying nanotechnologies to medicine opens novel and wide sources of funding opportunities within a general funding situation that is more and more limited. However, once funded the same culture of promise quickly demands results that show 'value for money': it asks for significant research activities and results instead of mere productivity. The longer the culture of nanotechnological promise remains more or less unredeemed the more pressing and daunting a most significant feature of the culture of promise becomes: The quest for fast science that demands from innovation processes the acceleration of reproducible outputs and applicable results. It seems obvious that the pressure to publish primarily 'positive results', as it is fostered by current research policy, has counterproductive effects on the most powerful tool scientists have: the experiment. However, good scientific practices cannot be separated from good experiments in order to produce reliable and reproducible results. Consequently, it is precisely the question of testing non/reproducibility and replicability, valuation of negative results, reliability and quality, which became a disputed issue of current biomedical and translational research and interrogates what is considered as 'good science' (see Luxenhofer, this issue [3]) [40-44]. The increasing pressure of publishing 'positive' and 'promising' results often veils the problems of emerging complexities of biomedical research rather than allowing an adequate time frame to understand them better and to grasp or solve relevant problems. The culture of promise enacts the perception and understanding of science which often downplays or even ignores that the work on emerging technologies goes hand in hand with a 'slowing down' of taken-forgranted practices and with the limits of given research tools. It overlooks that issues of nanomedicine set out problems first of all and that there is no 'double click' technology at hand to solve them without altering scientific practices and posing new questions and creating further issues [45]. It is the engagement with nanoscaled complexity that slows down the process by which scientist may possibly gain reliable and reproducible results. Thereby, scientists may lack the tools, instruments and techniques to create reliable data.

Nanomedical research asks for novel forms of assessing scientific practices and (nano-)objects involving the demanding process of characterization, questions concerning biodistribution, half-life prolongation, circulation time of novel drugs, controllability and sustainability of drug release, levels of intercellular concentration of nanodrugs and so on [46].The limits of taken for granted strategies, practices and technologies in the laboratory also includes for instance the questionability of highly taken for granted models in achieving valid results. So, for example, the production and use of model organisms (animal models) became a source of uncertainty and dispute in the process of accomplishing reliable results for research into human health issues $[47,48]$. At the same time, scientific practices cannot offer alternatives to animal models other than refuse them from an ethical point of view, which remains a minority position in science. Although the use of genetically engineered mouse models (GEMMs; e.g., transgenetic and/or humanized mice) is seen to speed up positive results, they become a concern of experimentation. In particular, it addresses the specific living context of animal models and questions the customized use of animal models being a mere genetically coded tool and obligatory desocialized matter of fact that is required to do adequate translational nanomedical research. The point I try to make is not that nanomedicine enforces a general verdict concerning animal models or any other problematic practice that involves living beings. But it raises the issue that for instance specific animal models like mice cannot be seen any more as taken-for-granted 'translational objects', in other words, as long established, normalized and standardized tools that promise good preclinical results for nanomedical research - small-sized organisms, more or less cheap, cost-efficient and ethically less problematic than bigger models [49-51]. Rather, it brings to the fore how biomedical research struggles and engages with understanding changing bodies - human and nonhuman alike - as experimental and situational objects of concern that are meant to translate into representative entities of each other. GEMMs (like humanized mice) for instance not only draw attention to similarities between the human and animal but also address the very uncertainties and insecurities of how different species relate with and are shaped by their environmental context as living and social entities: "For example, mouse groups may differ, despite being matched by genetics, supplier, gender, and age, in such intangible areas as nutrition, stress, circadian rhythm, etc." [42]. In consequence, translational biomedical research not only questions given understandings of different species, how they relate and how they represent each other. Bio- and nanomedical research also alter the relationship between animal models and human bodies, human and animal pathologies, bodies and their environment, the laboratory and the clinic, understandings of health and illness (see Meiners and Bölükbas, the second part of this Special Focus issue [52]) [50,53-55]. At the same time, the struggle of in vivo experimentation may give space for in vitro models to gain importance in innovating preclinical nanomedical research activities [54]. In all these cases, one 
has to recognize that biomedical research in general and nanomedicine in particular add social complexity that complicate already complex processes, objects, structures and their understandings.

Nanomedical research also alludes to emerging regulatory requirements, novel social effects on healthcare systems, changing understandings of illness and health, affordability and distribution of drugs as well as more general ethical obligations concerning emerging nanobiotechnologies, just to name a few. We said that with emerging technologies comes a change of organizational structures and work practices. Evidently, nanomedical research requires cross-disciplinary research, cross-disciplinary fora of discussion and publishing. It also requires cross-disciplinary peer-reviewing and transdisciplinary engagement, which demand new forms of communicating heterogeneous knowledge practices. The latter are still a novelty for modern discipline-driven research cultures [55] (for recent attempts to improve peer-reviewing, see, e.g., Public Library of Science (PLoS), eLife and EMBO Journal). Moreover, transdisciplinary engagement goes beyond the secluded space of the laboratory and assembles all relevant actors that are possibly affected by the specific nanomedical research projects.

\section{The laboratory at risk: experimentation \& the singularity of science}

Crucially, the quest for fast science, engendered by the culture of promise as outlined above, troubles an adequate engagement with unsolved and new problems of experimentation, underestimates failing experiments and negative results, disregards the creativity that stems from dealing with these unknowns and uncertainties and neglects the role of tacit knowledge and unintended surprises that may lead to novel issues and problems. Moreover, the lingering difficulty of reproducibility and reliability of experimental results troubles the mutual power relationship and social grain between researcher and research objects. What do I mean by that?

First of all, it alludes to a question that unites all the different disciplines of experimental sciences: Without reproducible and reliable data sciences have nothing discrete to offer. Thus, it endangers the singularity of science and the production of events through experimental settings that contribute to the changing of the history of humans and nonhumans [38-39,56-57]. The experimental setting can be understood as both a social and political arrangement by which human ideas and practices, technologies and nonhumans are associated in highly specific ways. The experimental setting is a social device, a mediating technology between human and nonhumans by which the researcher proposes novel ways of how on a small-scale processes and different entities come into being, relate, gain or change agency, alter their properties, in other words, function as transporters (e.g., for medical drugs) and/or transformers (e.g., as (nano-)medical drugs). Through the experimental setting, the researcher suggests specific modes of ordering which may work, work more or less or do not work at all. As Isabelle Stengers has put it:

"For scientists, it is actually a matter of constituting phenomena as actors in the discussion, that is, not only of letting them speak, but of letting them speak in a way that all other scientists recognize as reliable [58]. (...) The art of the experimenter is in league with power: the invention of the power to confer on things the power of conferring on the experimenter the power of speaking in their name." [59].

Only when it works in a reliable and reproducible way, the mutual power relationship between researcher and researcher is stabilized and enacts the singular power of science. Reliability in science is achieved when the respective data have withstood the possible objections by the scientific community that shares a concern for the data. In that moment, science - and this is what nanomedical research aims at - intervenes in the histories of humans and nonhumans and produces an 'event' by which novel forms of knowledge, processes and objects are created [39]. It is the ultimate aim of the scientist to create an 'event' that turns a speculative idea into a materialized form, into a novel, robust and reliable, small-scale social relation. Consequently, through the scientific event new (nonhuman) entities take part in human life and change its orderings. Once the experiment produces robust results, it silences all those who have disputed and questioned the visions and fictions of the experimenter so far. Within the 'culture of promise' such an event will be recognized as a starting point to pay off the fictions and visions that have been promoted and supported.

But, to be sure, the event itself does not decide its effects and trajectories beyond the specific laboratory experiment. All depends on how the event is perceived, discussed and judged by the scientific and wider community and how it offers possibilities to create new working experimentations and events which may - as in the case of nanomedicine - translate into preclinical and clinical testing, into an approved medical technology, a smart nanodrug or a novel diagnostic system. In order to be called a 'technology', nanomedical innovations, then, demand a complex association of events, which mark new settings, address different requirements and add social complexity. This process must also stand the test of being considered an adequate scientific innovation that extends beyond the lab, which necessarily involves scientific and nonscientific 
testing-agents (patients, clinicians, citizens, regulators, industry, philosophers, social and political scientists and so on).

\section{Lab experimentation as social \& political practices}

What makes the experimental setting in nanomedicine a social device is precisely the suggestion and composition of novel processes, associations and actors on a nanoscale. What makes the experimental setting a political device is the possibility of these nanoscale processes and objects to object to the proposed social relations as suggested by the experimenter. The experimental setting unfolds an open process by which it succeeds, fails or does not bring to the fore the suggested effects. The experiment names not only the singularity of science but experimenting also designates the very strength of the scientist. S/he proves to be a reliable and creative member of a political setting that experiments with and 'discusses' by the experimental setting how a specific social relation and related entities could look like and could act accordingly. Scientists constantly rethink and refine their experimental suggestions and accept as part of their daily work that experiments fail or are unsatisfying as well as they may produce unintended and surprising effects. Hence, the experimental setting provides a powerful location, a highly specified and controlled circumstance to engage with social and political processes. It reflects and creates a complex experimental sociopolitical situation.

Clearly, then, the experiment is a profound social and political device to engage with the nonhuman world. It frames the singularity of scientific engagement with the world and aims at the communication and solidarity between humans, things and nonhuman [60]. Good nanomedical experimental settings bring to the fore the surprising agency of nonhumans (e.g., nanoparticles, polymers, liposomes, siRNAs, etc.) in the way suggested by the experimenter through the experimental setting. The experimental setting gives space for novel agentic forces for which, if the experimentation works, the scientist becomes able to speak in their name.

It should be noted here that experimental interaction with nonhumans (model animals) is part and parcel of nanomedical research agendas. The more so we have to take care about the social complexity of nanomedical research and rethink the ways animal models gain agency in experimental settings and contribute to a creative experimental setting without abusing them or make them suffer. The lure of possibilities in experimental nanomedical work should always be under the constraint of hard work on relevance in order to do and care about good, responsible science.

\section{Intelligent experimentation}

It is most important, the more so if living systems are involved and the problems set out by nanomedicine, to recognize the "necessity for an intelligent experimentation, which assumes the risky responsibility of asking relevant questions" [61]. Intelligent experimentation is precisely what is required to care for the social complexity in nanomedical research. It addresses the responsibility of the researcher to engage in speculative experiments, which may have significant consequences for vulnerable animal and human actors involved within and beyond the specific laboratory settings. Moreover, experimental practices name risky endeavors since the experimental situation and the processes and entities involved may prove the scientist's speculation as unfeasible. One also has to note that intelligent experimentation is not so much about inquiries into generalities, but emerge from a well-defined situation that remains nevertheless unsolved (e.g., the development of targeted nanodrugs for a specific cancer or other diseases) and thus names a socially most complex and high-risk engagement.

To assume relevant questions in nanomedical research also means to involve all relevant actors and move beyond the secluded space of the laboratory to sharpen or question the relevance of the problem and how it is framed by the experiment. Clearly, experimental lab processes are quite different than controversies outside the laboratory. It thus requires extending the controversies, propagating the social and political processes and altering the means of disputing and communicating. Beyond the laboratory space, scientific experimentation is one of many forms of intelligently experimenting with complex social situations. There are two possibilities of extending intelligent experimentation beyond the lab that I will further discuss: 'public expert controversies' and 'publics'. Both options offer modes of caring for the social complexity of nanomedical research outside the lab but are necessarily entangled with the practices and objects of lab experimentation.

\section{Public expert controversies}

I have argued so far that to care about social complexity is to engage with an emerging situation for which no solution is given, but which may unleash unintended risks. Hence, complex situations require a careful consideration of relevant questions. I also argued for understanding the laboratory experiment as a vital social and political device to care about social complexity in nanomedicine and how it is troubled by the requirements of fast science. As a center problem, I tackled the ambiguous relationship between the culture of the nanotechnological promise and social complex- 
ity of nanomedical research issues. This asymmetry is also at the heart of a prominent public controversy in 2003/2004 between EK Drexler and RE Smalley [6265]. Drexler, former MIT engineer and IT futurologist whose books 'Engines of Creation: The Coming Era of Nanotechnology' (1986) and 'Nanosystems: Molecular Machinery, Manufacturing and Computation' (1992) were most important in popularizing nanotechnology and its promises of radical societal change with technoscientific means. Smalley was a chemist and Noble Prize laureate as well as a major figure in the nanotechnological initiatives in the USA for many years.

\section{Theoretical visions \& practitioners}

The debate between Drexler and Smalley not only focuses on the vision of self-assembling nanorobots but also draws attention to the emergence of a publiccontroversy that complicates the promise of nanotechnology. Drexler's argument reads like this:

“(...) Replicating assemblers will copy themselves by the ton, then make other products such as computers, rocket engines, chairs, and so forth. They will make disassemblers able to break down rock to supply raw material. They will make solar collectors to supply energy. (...) Assemblers will be able to make virtually anything from common materials without labor, replacing smoking factories with systems as clean as forests." [66].

Applied to medicine, Drexler stresses that "cell repair machines will bring a fundamental breakthrough: they will free medicine from reliance on selfrepair as the only path of healing" [67]. With the help of nanoassemblers, so the promise of an engineer's vision, virtually everything is possible and everything translates - without human labor and the necessity of human control - into the desired objectives, including so-called 'engines of healing' that promise a life without disease, presupposing that the atomic manufacture is correctly assembled. "Molecular nanotechnology assemblers", so Drexler, "would change society's relationship to molecules and matter as fundamentally as the computer changed our relationship to bits and information" [64]. Drexler's 'nano'-narrative offers a theory of relationships of smallest, basic units (minimum of two atoms), which is meant to authorize global practical applications. It allows Drexler, the engineer and his theoretical assumptions that reflect a strong link with physics rather than chemistry, to imagine and promise a multiplicity of nanoengineered futures that also translate into nanomedical futures assembling 'engines of healing'. In a short but intriguing statement Smalley, the chemist, denies the possibility of Drexler's nanoassemblers: "How soon will we see the nanometer-scale robots envisaged by Drexler and other molecular nanotechnologists? The simple answer is never." [68].

The debate not only reflects a vivid dispute between the different specific arguments of Drexler and Smalley, but also brings to the fore a controversy over basic principles of molecular complexity, what argument can be considered scientific, what argument appears as feasible, what can we expect from nanotechnologies and so forth. The controversy was not closed by an agreement over issues but rather remains a discussion over concerns perceived from different disciplinary positions that seem to be incompatible to contribute to a solution. To be sure, and this is the point of public expert controversies, controversies like the Drexler/Smalley debate keep the nanoproblem alive by adding social complexity; they do not need consensus. Public expert controversies open up a space for debating the relevance of each argument and allowing space for the possibility to alter the arguments presented, of generating relevant questions and related problems. Public expert controversies are risky adventures. They are sterile and unproductive, if the experts are not willing to take risks and seal off their position from being challenged. Scientists are experienced with taking similar risks. They take risks in the laboratory where they are constantly questioned by the experimental process and the objections of nonhuman configurations. So it should be not very problematic to take risks in public controversies as well! However, nanomedical research misses public expert controversies. So, dear scientists, make your controversies public!

\section{Multiscaled social complexity}

What is interesting about Smalley's critical statement is not so much his straightforward denial of Drexler's futurist vision. Rather, Smalley refers to issues of social complexity in a highly intriguing way when he links the nanoworld with the macroworld. Smalley says:

"When a boy and a girl fall in love, it is often said, that the chemistry between them is good. This common use of the word 'chemistry' in human relations comes close to the subtlety of what actually happens in the more mundane coupling of molecules. In a chemical reaction between two 'consenting' molecules, bonds form between some of the atoms in what is usually a complex dance involving motion in multiple dimensions. Not just any two molecules will react. Chemistry is subtle. (...) You don't make a girl and a boy fall in love by pushing them together. (...) Like the dance of love, chemistry is a waltz with its own step-slide-step in three quarter time. Wishing that a waltz were a merengue - or that we could set down each atom in just the right place - doesn't make it so." [63].

Smalley's contrasting view to Drexler's nanotechnological promise-land highlights his insistence of the 
molecular world as a complex social world of highly specific, affective processes and relations, which enact a multiplicity of diverse effects. For Smalley, the empirical is too complex to think that everything is or could be possible. By referring to the complex social interactions that may create the more or less exceptional situation where molecules are bonding, so that they may (or not) work in the way they are suggested to do so, Smalley translates Drexler's theoretical vision into issues of a practitioner.

Whereas Drexler's visions have been important in popularizing the fictive potentialities of nanomachines and its application to healthcare issues, Smalley shifts the context from theoretical visions to the laboratory of chemical experimentation. Smalley's translation of Drexler's visions offers an interesting possibility of intelligent experimentation inasmuch as his position translates Drexler's exciting ideas into the constraints of a laboratory situation of a chemist without referring to the lab experiment as such, but to the complexities of bonding, in other words, of social processes in the macroworld. Smalley enriches the networks of explanation by switching scale.

What Smalley makes us aware that to engage in public expert controversies conceives nanomedical research as a complex set of experimental practices, which - under conditions of a more or less uncertain and often unknown nanoworld - aim at relations of 'good chemistry'. Clearly, then, the sound and sustainable medical nanotechnology is only the rare endproduct, the materialized form of a multiplicity of emerging relations, the experiences of a nonlinear and often highly disputed research process, of experimental failures and successes, of unexpected results and so on. Like falling in love in the macroworld, chemical bonding in the molecular world unfolds an eventful relation, which translates what has been related into a new and highly significant social complex. And as it is with falling in love, eventful bonding relations in nanoresearch are exceptional events. These events may happen everyday day but they are not everyday events.

Engaging public expert controversies alludes to the idea of enabling and organizing possibilities to create a process of affecting and being affected by a complex situation, and to pose problems for which the actors involved have no ready-made solutions. Like the scientific experiment, it articulates the need to cultivate bonding experiments (as striving as they may be) by which different practices and actors become capable to affect each other and gain agency through the interaction with the respective other. Clearly, the bonding practices in public expert controversies are not about falling in love nor is it merely about molecular bonding. It nevertheless translates the idea of a complex form of interaction between otherwise nonrelated actors that come together and give importance in assembling a specific problem of concern. By this translation and shift, the secluded lab experiment as well as the theoretical speculation translates into a public event. Thereby, a socially complex issue like 'nanotechnology' or 'nanomedicine' becomes a shared concern that is publicly accessible and disputable. Consequently, public expert controversies aim at an extended communication between relevant actors, who engage in different practices, and who may locate the problem and issues in differently (e.g., not in theory or in the lab as Drexler and Smalley, but, e.g., in the human body as patients and clinicians do).

I have argued that the scientific experiment and the public expert controversy are risky endeavors insofar as the involved actors may object to the other's position and/or may even change their position accordingly. Given the experiences from the lab, the scientist should not see such risks as a situation that needs to be avoided or perceived as irrelevant to or threatening scientific work. Every scientist has profound experience of experimental settings. In the lab, scientists constantly are confronted with experimental objects, which may not give consent to the experimental setting of the scientist and in return may change how the scientist is thinking, understanding and engaging with these objects [69]. To be sure, like Smalley's process of bonding molecules, public experimentations are not simply about the mechanical placing of given and exchangeable objects and positions as Drexler's nanoassemblers suggest. Rather, public experimentation like chemical bonding - implicate 'a complex dance involving motion in multiple dimensions', which link the secluded space of the scientific laboratory and create a novel, but similarly precious, public laboratory of nanomedical research.

Concluding, I suggest that Smalley's complicated and rather slow processes of bonding molecules and nonconsensual public expert controversies need to be translated into publics. It also means that visions like Drexler's nanofictions should not be discarded from expert controversies and public engagement. Publics of engagement name events where all the different actors, disciplines and groups concerned with the issue, create and dispute - case by case - the particular conditions of possibility of nanomedical in(ter)vention.

\section{Publics}

So far I have argued that processes of translation in nanomedical research do not complicate complexity as a newly added feature does complicate the mechanic system of, for example, a watch. Rather, the translation from ideas into preclinical in vitro testing into in vivo 
models into clinical testing and into approved usage in humans constantly adds social complexity (novel questions and problems, unexpected effects (shortand long-term), reflexive loops of translation, etc.). The very discovery of the nanoeffect that scientists try to appropriate in medical research is a good example to illustrate my point: a difference in size may produce unforeseeable properties and effects of an object in relation to its environment. Hence, the nanoeffect draws our attention and awareness to a social phenomenon. It names a social phenomenon inasmuch as it reconfigures the properties and agencies of objects by recreating the relations of interactions that affect these properties. It also opens possibilities for new issues that gain relevance in biomedical research, which may affect the healthcare system.

\section{Extending the social in nanomedicine}

To aim at 'good chemistry' in nanomedical research, one needs to extend social relations to connect the secluded spaces of the lab with nonsecluded forms of public experimentation. In that sense, public labs should unfold a nonthreatening space where the different (and often vulnerable and uneven powerful) concerned actors can meet and discuss and shape their different positions, address their uncertainties and problems, state their interests, unpack their experiences, interpretations and perceptions, name their expectations, hopes, anxieties and so on. It creates a space where these different perspectives of a shared matter of concern (diagnosis/treatment of an illness) circulate between the different scientific practices, research groups and disciplines, to and from science, to and from the clinic, doctors, patients and patient organizations, to and from differently concerned citizens, to and from legal and ethical concerns, to and from industry and so on. Publics are spaces for novel forms of research, spaces where science is in the making and not so much where experts and laypersons meet or stakeholders and disciplines exchange their predefined positions!

Nanomedical publics bring together people (and their practices and objects) who share a concern for an issue for which no immediate answers and fixed solutions are given (or can be expected) [70]. Publics, then, engage with the ambiguities and uncertainties of scientific research practices, their relevant objects of concern and their possible effects inside and outside the scientific laboratory. The different actors of publics share, engage and express a 'sense of importance' of nanomedical research, how it affects and connects the different scales of living systems (nano, micro, macro), how it produces different but shared concerns of translating scientific ideas into laboratory experiments into possible objects and technologies of healthcare (therapeutic and diagnostic) [71].

\section{Slowing down the culture of promise}

In particular, nanomedical publics interrogate the 'culture of promise' concerning emerging nanotechnologies. It puts to the public test novel research agendas like nanomedical research for which it remains an open question if and how certain technologies may evolve or may not and if they are able to improve current technologies of healthcare. Publics subject scientific research to a reality test, which - as it is in the case of nanomedicine - is in need to prove its attributed role of being a key actor in innovating healthcare by providing novel technologies of diagnosis and treatment [72]. Problem-oriented publics offer the possibility to disentangle the complexities of everyday nanomedical research from the global and all-too abstract discourse on generic terms such as 'nano' or 'nanotechnologies'. By making specific nanomedical concerns public, publics focus on and engage with the practices, questions and uncertainties as they emerge from scientific experimentation that is committed to good scientific practices.

With this proposed shift, I like to underline that the time is more than ripe to untie the close intertwining between the general, fast science' promises of 'nano' or 'nanotechnology' and nanomedicine as its application. Publics, so my argument, can be seen as a productive way of questioning, discussing, disputing and complexifying the excessive 'culture of promise' concerning nanotechnologies. Making nanomedical research public can be considered as an attractive democratic remedy to avoid the vicious trap between positive and negative overhype, which seems to be symptomatic for an excessive economy of promises that drives the popular discourse of nanotechnology. Moreover, publics interrogate the stark division between 'secluded research' (lab science) and 'open politics' (society), 'experts' and 'laypersons', 'facts' (scientific) and 'values' (societal) [73]. These divisions prove to be highly abstract, unrealistic and unhelpful divisions once one engages with the concrete nanomedical research process itself. The more so if research is thought to have significant effects on vulnerable human life situations. Publics can be understood as an upgraded and extended enquiry into the social complexity of nanomedical and biomedical research. It will necessarily slow down the process of current 'fast science' research policies and structures [74]. Slow science is a plea for a good chemistry, which takes time, and, following Smalley, unfortunately does not translate easily from a waltz to a merengue.

This is the point I try to make: As this special issue lucidly shows, current nanomedical research is not 
primarily about the mechanistic scaffolding of molecules and the application of nanotechnologies (that is accustomed and well-functioning, robust and sustainable technologies). But it is the ambitious engagement with highly unknown processes and effects that refer to the social complexity of experimental objects in the making, which require innovative (but often nonaccustomed) and risky research practices and structures.

\section{Connecting lab \& field experimentation}

Publics do not exist as a standing-reserve and should not be conflated with the general public. Rather, publics need to be created, put into practice around a specific matter of concern and its multiple requirements [75-77]. Publics also differ significantly from attempts of 'public understanding of science', whereby the scientific expert meets the layperson to inform and discuss his/her research in order to possibly gain recognition and approval for given research strategies. Publics do not consist primarily of 'experts' who have superior access to knowledge that needs to be distributed and acknowledged by the nonscientific other (patients, laypersons, concerned citizens, clinicians, policy makers). Rather, publics emerge (and need to be organized) under conditions of shared uncertainty. In that sense, there are no experts and there is no clear divide that separates off the expert from the layperson. Consequently, publics do not promote a hierarchy of expertise and knowledge. Or differently put, publics are highly demanding events since they bring together the different ideas, perceptions and practices of relevant actors (e.g., the clinic, patients, interested citizens) that may dispute and alter those of the respective other in order to complexify the social importance of the problem of concern.

Publics are measured by the multiple ways of initiating the crossings between the different perspectives and actors and how these crossings unfold novel ways of engaging with the object of concern, of sharpening the relevant questions and framing further research trajectories. Clearly, publics are most vital in engaging with emerging research agendas and technologies (like nanomedical research) since there is no 'double click' that smoothly and mechanically connects ideas with research practices and applications. Publics do not merely discuss or distribute fixed and stable forms of scientific knowledge [45]. On the contrary, publics contribute to scientific research in the making. Publics practice science. Publics unfold 'science in action' [39].

Obviously, publics engage experiments with other means than those established in the laboratory. To be sure, this does not mean that the very singularity of science and its very strength - the laboratory experiment - does not play a vital role in publics. The laboratory plays a most attractive model precisely in understanding the idea of nanomedical publics and ideas of social and political experimentation as developed in this paper. This is the more important since the power relations of the laboratory experiment allow to unfold novel and stabilized forms of social relations (e.g., a possible diagnostic agent, a polymeric drug for cancer treatments). These innovations, then, may become powerful configurations for extended social relations outside of the laboratory, in and outside of the clinic and may contribute to improved healthcare settings and practices. This highly distributed social complexity of nanomedical research requires distributed forms of social and political experimentation.

Publics initiate an experiment that aims at a social and political process in as much as it assembles a network of concatenation of highly different entities, perspectives and practices, contested objects, fictions, expectations, resistances, prejudices and so forth. Importantly, there is no linear trajectory to be easily followed that smoothly moves from lab experimentation to field experimentation. Nor is there a predictable increase or decrease of complexity regarding the different forms of experimentation. Rather, what we find is the mutual translation of a set of complexities and activities, more or less known, into emerging and different sets of complexities and activities more or less known moving from in vitro to in vivo, from discipline to discipline, from labs to labs, from nano-, micro- to macro-scales, from labs to fields, from one body to another, from healthy to ill bodies and so on.

\section{Conclusion}

Publics initiate and connect lab experiments of sciences and field experiments of nanomedical research that go beyond the lab. Publics create centers of communicating the social complexity of translational medicine as they emerge from lab experiments with nonhumans, objects and configurations and translate into clinical and wider field experiments including human life and its environment. To sustain the well-being of nanomedical research, these translations need to be taken into account in order to safeguard the effects of new nanomedical objects and medical technologies. It requires a culture of care of 'slow science'. It offers a most vital contrast that tames the culture of promise of 'fast science'. Thus, as a matter of caring about good scientific practices, nanomedicine needs to celebrate and bring together the diverse controversies and relevant actors as they emerge in the lab, in public expert controversies and publics. 


\section{Future perspective}

Caring for social complexity in nanomedicine names a vital requirement for the future of its well-being. This includes a close network of experimentation that links the issues and problems posed by the laboratory with public expert controversies and publics. To engage in these new forms of doing and caring about science and its relevant contexts requires the cultivation of these diverse democratizing tools of nanomedical research. As the cross-disciplinary workshop 'Quo vadis nanomedicine?' (April 2014/Exeter, UK) has shown, scientists start to realize the importance of these new forms of experimentation and acknowledge that the engagement with relevant actors in the broad field of nanomedicine is a precondition to ask and investigate relevant questions and problems [78]. Having said this, current nanomedical science needs more opportunities to cultivate these new modes of caring, since public engagement as outline here remains new and unpracticed territory for many scientific actors.

\section{Financial \& competing interests disclosure}

This paper is supported by a 5 -year research project 'Innovations in Nanomedicine. Object- and Knowledge-Formation in Cross-Disciplinary Scientific Practices' (Schumpeter Fellowship 2010-2015) funded by the German VolkswagenStiftung. The author has no other relevant affiliations or financial involvement with any organization or entity with a financial interest in or financial conflict with the subject matter or materials discussed in the manuscript apart from those disclosed.

No writing assistance was utilized in the production of this manuscript.

\section{Open access}

This work is licensed under the Creative Commons Attribution 4.0 License. To view a copy of this license, visit http://creativecommons.org/licenses/by/4.0/

\section{Executive summary}

- Nanomedical research agendas require a method of caring about the complexity of nanomedical research projects for which no solution is given or can be expected in the nearest future.

- Caring about nanomedical complexity requires new forms of experimentation.

- Three possibility of experimentation have been discussed:

- Laboratory experiment and scientific analysis;

- Public expert controversies;

- Publics.

- These modes of experimentation offer a taming contrast to the excessive and counterproductive 'culture of promise' of nanotechnologies.

\section{References}

1 Deleuze G, Guattari F. What is Philosophy? Verso Books, NY, USA (1994).

2 Barz M. Complexity and simplification in the development of nanomedicines. Nanomedicine (Lond.) 10 (20), 3093-3097 (2015).

3 Luxenhofer R. Polymers and nanomedicine: considerations on variability and reproducibility when combining complex systems. Nanomedicine (Lond.) 10(20), 3109-3119 (2015).

4 Guchet X. What's in a word? The person of personalized (nano) medicine. Nanomedicine (Lond.) 10(20), 3167-3179 (2015).

5 Pierce R. Translational nanomedicine through the therapeutic window. Nanomedicine (Lond.) 10(21), doi:10.2217/NNM.15.168 (2015) (In press).

6 Ach JS, Lüttenberg B. Nanobiotechnology, Nanomedicine and Human Enhancement. Lit-Verlag, Berlin, Germany (2009).

7 Allhoff F, Lin P, Moor J, Weckert J. Nanoethics. The Ethical and Social Implications of Nanotechnology. Wiley, NJ, USA (2007).

8 Allhof F, Lin P. Nanotechnology \& Society. Current and Emergent Ethical Issues. Springer, NY, USA (2009).

9 Schmid G, Brune H, Grünwald A et al. Nanotechnology. Assessment and Perspective. Springer, Berlin, Germany (2006).
10 Kaiser M, Kurath M, Maasen S, Rehmann-Sutter CH. Governing Future Technologies. Nanotechnologies and the Rise of an Assessment Regime. Springer, Dordrecht, The Netherlands (2010).

11 Kjolberg K, Wickson F (Eds). Nano Meets Macro: Social Perspectives on Nanoscaled Sciences and Technologies. Pan Stanford, Singapore (2010).

12 Schillmeier M. What ELSA/I makes big and small in nanotechnological research. In: Absence in Science, Security and Policy. From Research Agendas to Global Strategy. Rappert B, Balmer B (Eds). Palgrave Macmillan, Basingstoke, UK, 55-77 (2015).

13 Schummer J, Baird D. Nanotechnology Challenges. Implications for Philosophy, Ethics and Society. World Scientific, NJ, USA (2006).

14 Latour B. Reassembling the Social. An Introduction to ActorNetwork Theory. Oxford University Press, Oxford, UK (2005).

15 Schillmeier M. Eventful Bodies. The Cosmopolitics of Illness.Ashgate, Farnham, UK (2014).

16 Schillmeier M, Domènech M. New Technologies and Emerging Spaces of Care. Ashgate, Farnham, UK (2010).

17 Puig de la Bellacasa M. Matter of care in technoscience: assembling neglected things. Soc. Stud. Sci. 41(1), 85-106 (2011). 
18 Latour B. An Inquiry into Modes of Existence. An Anthropology of the Moderns. Harvard University Press, MA, USA, 46 (2013).

19 Latour B. An Inquiry into Modes of Existence. An Anthropology of the Moderns. Harvard University Press, MA, USA, 58 (2013).

20 Stengers I. Power and Invention. Situating Science. University of Minnesota Press, MN, USA, 12 (1997).

21 Serres M. Hermes IV Verteilung. Merve, Berlin, Germany (1993).

22 Ge Y, Li S, Wang S, Moore R. Nanomedicine. Springer, NY, USA (2014).

23 Webster TJ. Nanomedicine. Technologies and Applications. Woodhead, MS, USA (2012).

24 Tibbals HF. Medical Nanotechnology and Nanomedicine. CRC Press, FL, USA (2011).

25 Jain KK. The Handbook of Nanomedicine. Humana Press, NJ, USA (2010).

26 Malsch I, Emond C. Nanotechnology and Human Health. CRC Press, FL, USA (2014).

27 Roco MC, Bainbridge WS. Converging Technologies for Improving Human Performance. Nanotechnology, Biotechnology, Information Technology and Cognitive Science. Kluwer, Dordrecht, The Netherlands (2003).

28 Alexiou C (Ed.). Nanomedicine - Basic and Clinical Applications in Diagnostic and Therapy. Karger, Erlangen, Germany (2011).

29 Nordmann A. Enhancing material nature. In: Nano Meets Macro: Social Perspectives on Nanoscaled Sciences and Technologies. Kjolberg K, Wickson F (Eds). Pan Stanford, Singapore, 283-303 (2010).

30 U.S. Department of Health and Human Services. National Institutes of Health, National Cancer Institute Cancer Nanotechnology. Going Small for Big Advances. Cancer Diagnosis, Prevention, and Treatment. NIH Publication, MD, USA, 1 (2004).

31 Freitas R. Nanomedicine. Volume I: Basic Capabilities. Landes Bioscience, TX, USA, 17-18 (1999).

32 Etheridge ML, Campbell SA, Erdman AG, Haynes CL, Wolf SM, McCullough J. The big picture on nanomedicine: the state of investigational and approved nanomedicine products. Nanomedicine (Lond.) 9(1), 1-14 (2013).

33 Hehenberger M. Nanomedicine: Science, Business, and Impact. Pan Stanford, Singapore (2015).

34 Igarashi E. Nanomedicines and Nanoproducts: Applications, Dispositions, and Toxicology in the Human Body. CRC Press, London, UK (2015).

35 Jain KK. The Handbook of Nanomedicine (2nd Edition). Humana Press, NJ, USA (2012).

36 Kostaleros $\mathrm{K}$. The emergence of nanomedicine. Nanomedicine (Lond.) 1(1), 1-3 (2006).

37 Whitehead AN. Science and the Modern World. The Free Press, NY, USA (1967)

38 Stengers I. The Invention of Modern Science. University of Minnesota Press, MN, USA (2000).

39 Latour B. Science in Action. How to Follow Scientists and Engineers Through Society. Harvard University Press, MA, USA (1987).
40 Drummond C. Replicability is not reproducibility: nor is it good Science. Presented at: Evaluation Methods for Machine Learning Workshop at the 26th ICML. Montreal, Canada, 14-18 June 2009.

41 Kraus WL. Do you see what I see? Quality, reliability, and reproducibility in biomedical research. Mol. Endocrinol. 28(3), 277-280 (2014).

42 Casadevall A, Fang FF. Reproducible science. Infect. Immun. 78(12), 4972-4975 (2010).

43 Bissell M. Reproducibility: the risks of the replication drive. Nature 503, 333-334 (2013).

44 Trouble at the lab. The Economist. www.economist.com/news/briefing

45 Latour B. An Inquiry into Modes of Existence. An Anthropology of the Moderns. Harvard University Press, MA, USA (2013).

46 PourGashtasbi G. Nanotoxicology and challenges of translation. Nanomedicine (Lond.) 10 (20), 3121-3129 (2015).

47 Seok J, Warren HS, Cuenca AG et al. Genomic responses in mouse models poorly mimic human inflammatory diseases. Proc. Natl Acad. Sci. USA 110(9), 3507-3512 (2013).

48 Perrin S. Preclinical research: make mouse studies work. Nature 507, 423-425 (2014).

49 Michael M, Wainwright S, Williams C. Temporality and prudence: on stem cells as "phronesic things". Configurations 13, 373-394 (2005).

50 European Commission. Of mice and men - are mice relevant models for human disease? http://ec.europa.eu/research/health/pdf/summary

51 Birke L. Animal bodies in the production of scientific knowledge: modelling medicine. Body Soc. 18, 156-178 (2012).

52 Meiners S, Bölükbas DA. Lung cancer nanomedicine: potentials and pitfalls. Nanomedicine (Lond.) 10 (21), doi: 10.2217/NNM.15.155 (2015) (In press).

53 Davies G. What is a humanized mouse? Remaking the species and spaces of translational medicine. Body Soc. 18, 126 (2012).

54 Leonard F, Collnot E, Lehr CM. A three-dimensional coculture of entercytes, monocytes and dendritic cells to model inflamed intestinal mucosa in vitro. Mol. Pharm. 7(6), 2103-2119 (2010).

55 Kuhn TS. The Structure of Scientific Revolutions. University of Chicago Press, IL, USA (1970).

56 Latour B. The Pasteurization of France. Harvard University Press, MA, USA (1988).

57 Schillmeier M. Science, cosmopolitics and the question of agency. Kant's Critique and Stenger's Event. In: Agency Without Actors? Rethinking Collective Action. Passoth JH, Peuker B, Schillmeier M (Eds). Routledge, NY, USA, 31-53 (2012).

58 Stengers I. Power and Invention. Situating Science. University of Minnesota Press, MN, USA, 85 (1997).

59 Stengers I. Power and Invention. Situating Science. University of Minnesota Press, MN, USA, 165 (1997).

60 Serres M. Hermes I. Kommunikation. Merve, Berlin, Germany (1991). 
61 Stengers I. Power and Invention. Situating Science. University of Minnesota Press, MN, USA, 17 (1997).

62 Drexler KE. Machine-phase nanotechnology. Sci. Am. 285(3), 74-75 (2001).

63 Smalley RE. Of chemistry, love, and nanorobots. Sci. Am. 285(3), 76-77 (2001).

64 Drexler KE, Forrest D, Freitas RA et al. On physics, fundamentals, and nanorobots: a rebuttal to Smalley's assertion that self-replicating mechanical nanorobots are simply not possible. Institute for Molecular Manufacturing (2001). www.imm.org/publications/sciamdebate2/smalley/

65 Baum R, Drexler E, Smalley R. Nanotechnology: Drexler and Smalley make the case for and against 'molecular assemblers'. C $2 E N$ 81(48), 37-42 (2003).

66 Drexler KE. Engines of Creation: The Coming Era of Nanotechnology. Anchor Books, NY, USA, 63 (1986).

67 Drexler KE. Engines of Creation: The Coming Era of Nanotechnology. Anchor Books, NY, USA, 106 (1986).

68 Smalley RE. Of chemistry, love, and nanorobots. Sci. Am. 285(3), 76 (2001).

69 Rheinberger HJ. Towards a History of Epistemic Things: Synthesizing Proteins in the Test Tube. Stanford University Press, Singapore (1997).

70 Dewey J. The Public and Its Problems. An Essay in Political Inquiry. Penn State University Press, PA, USA (2012).
71 Whitehead AN. Modes of Thought. The Free Press, NY, USA (1968).

72 White Paper to the Horizon 2020 Framework Programme for Research and Innovation - recommendations from the nanomedicine community, ETP nanomedicine (2013). www.etp-nanomedicine.eu/public/press-documents

73 Callon M, Lascoumes P, Barthe Y. Acting in an Uncertain World. An Essay on Technical Democracy. MIT Press, MA, USA (2001).

74 Stengers I. Another science is possible. A plea for slow science. Inangural Lecture Chair Willy Calewaert. Brussels, Belgium, 13 December 2011.

75 Marres N. Issues spark a public into being. A key but often forgotten point of the Lippmann-Dewey debate. In: Making Things Public: Atmospheres of Democracy. Latour B, Weibel P (Eds). MIT Press, MA, USA (2005).

76 Marres N, Rogers R. Recipe for tracing the fate of issues and their publics on the web. In: Making Things Public: Atmospheres of Democracy. Latour B, Weibel P (Eds). MIT Press, MA, USA (2005).

77 Latour B, Weibel P. Making Things Public: Atmospheres of Democracy. MIT Press, MA, USA (2005).

78 Schillmeier M, Luxenhofer R, Barz R. Quo vadis nanomedicine? Nanomedicine 9(14), 2083-2086 (2014). 